\title{
Presencia y Morfometría de Forámenes y Canales en Relación a las Espinas Mentonianas
}

\author{
Presence and Morphometry of Foramina and Canals in Relation to Mental Spines \\ Reinaldo Soto; Felipe Cáceres \& Roberto García
}

SOTO, R.; CÁCERES, F. \& GARCÍA, R. Presencia y morfometría de forámenes y canales en relación a las espinas mentonianas. Int. J. Morphol., 30(2):417-421, 2012.

RESUMEN: Los procedimientos quirúrgicos desarrollados en la porción anterior del cuerpo mandibular, como por ejemplo la instalación de implantes oseointegrados, genioplastias, profundizaciones de flanco lingual, fracturas, etc. pueden presentar complicaciones de importancia cuando estas invaden estructuras vasculares, con la consecuente hemorragia y formación de hematomas sublinguales. Dentro de estos elementos anatómicos se encuentran forámenes y canales que se ubican en relación a las espinas mentonianas. El objetivo de este estudio fue cuantificar la ubicación, diámetro y longitud de los forámenes y canales presentes por superior, inferior y entre las espinas mentonianas superiores e inferiores en 71 mandíbulas secas de la Unidad de Morfología de la Universidad de los Andes las que fueron fotografiadas. Analizando las fotografías con el programa Photoshop® Cs 3 extended, se midieron en pixeles el diámetro y longitud de los forámenes y canales para luego transformarlos en mm. El 97\% de las mandíbulas observadas presentaron al menos un foramen, el 45\% presentó dos y el $31 \%$ tres. En relación a su ubicación el $82 \%$ de las mandíbulas presentó forámenes por superior a las espinas y el 76\% por inferior. El diámetro promedio fue de $0,73 \mathrm{~mm}$ para los de ubicación superior y de $0,71 \mathrm{~mm}$ para los de ubicación inferior. La longitud promedio de los canales fue de $6,77 \mathrm{~mm}$ para los ubicados por superior y de 5,5 $\mathrm{mm}$ para los de ubicación inferior a las espinas mentonianas. Debido a la alta prevalencia de forámenes y canales en nuestra muestra es recomendable tenerlos siempre en cuenta en la planificación de intervenciones quirúrgicas que involucren ya sea el flanco lingual mediano mandibular o el cuerpo mandibular en la zona de la sínfisis.

PALABRAS CLAVE: Canal lingual; Espinas mentonianas.

\section{INTRODUCCIÓN}

Los procedimientos quirúrgicos desarrollados en la porción anterior del cuerpo mandibular, como por ejemplo la instalación de implantes oseointegrados, genioplastias, profundizaciones de flanco lingual, fracturas, etc. pueden presentar complicaciones de importancia cuando estas invaden estructuras vasculares, con la consecuente hemorragia y formación de hematomas sublinguales. Estos pueden llevar al paciente a tener incluso riesgo vital al ocasionar una obliteración de la vía aérea (Isaacson, 2004; Kalpidis \& Setayesh, 2004) causada por el acúmulo de sangre en la región sublingual, submandibular y submentoniana, provocando la elevación y movimiento hacia posterior de la lengua.

Diversas estructuras neurovasculares suelen abordar la mandíbula por múltiples forámenes, los cuales no son descritos en los tratados de anatomía clásica. El origen de estos vasos y nervios que ingresan a la mandíbula no está del todo claro. Flanagan (2003) describe que las principales arterias que participan en la irrigación del sector mandibular anterior corresponden a ramos de las arterias lingual, facial (ramas directas de la carótida externa) y la arteria alveolar inferior (rama inferior de la arteria maxilar). McDonnell et al. (1994) plantea que los elementos que penetran estos forámenes son principalmente vasculares y dependiendo de la altura en que se encuentren pueden ser derivados de la arteria sublingual (rama terminal de la arteria lingual), en el caso de tratarse de forámenes superiores a las espinas mentonianas inferiores o derivados de la arteria submentoniana (rama colateral de la arteria facial), si se trata de forámenes inferiores a las espinas mentonianas inferiores.

Se ha visto en estudios descriptivos que dentro del sector anterior mandibular, los forámenes ubicados en la línea mediana en relación a las espinas mentonianas son los más constantes y de mayores dimensiones (McDonnell et al.; Lagraña et al., 2006; Tagaya et al., 2009). Es por esta razón que se decidió estudiar en población chilena la prevalencia de los forámenes, el tamaño de estos y la longitud de los canales ubicados en relación a las espinas mentonianas. 


\section{MATERIAL Y MÉTODO}

Se seleccionaron 71 mandíbulas humanas secas, con sexo y edad indeterminada, de la Unidad de Morfología de la Universidad de los Andes. Estás fueron enumeradas con un lápiz de tinta permanente en la región de la fosita sublingual izquierda de forma aleatoria desde el número 1 hasta el 71. Se desarrolló una tabla de datos

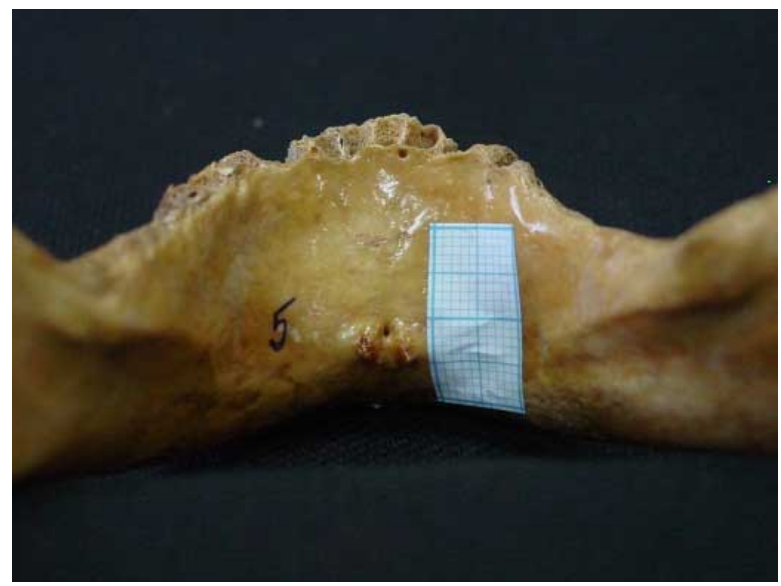

Fig. 1. Identificación de cada mandíbula.

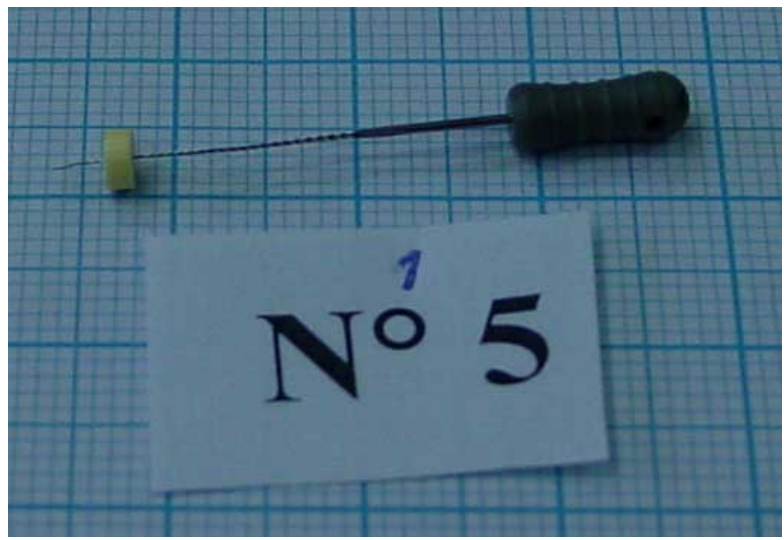

Fig. 2. Traslado de la medición de longitud a papel milimetrado.

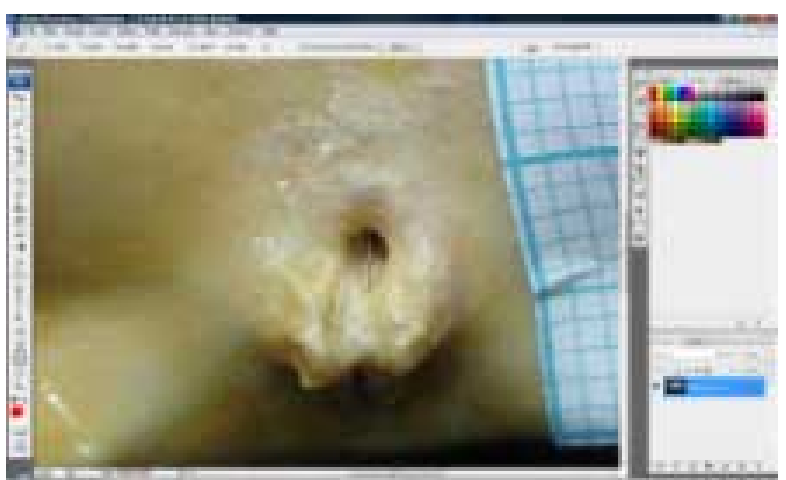

Fig. 3. Medición de diámetros de forámenes con programa Photoshop ${ }^{\circledR}$ Cs 3 Extended. con el programa Excel® donde se anotaron correlativamente los siguientes datos: número de piezas remanentes, número de piezas remanentes en el grupo $\mathrm{V}$, número de forámenes en la línea media, número de forámenes superiores a las espinas mentonianas superiores, número de forámenes entre las espinas mentonianas superiores, número de foramen entre las espinas mentonianas superiores e inferiores, número de forámenes entre las espinas mentonianas inferiores, número de forámenes inferiores a las espinas mentonianas inferiores, diámetro correspondiente a cada uno de los forámenes descritos anteriormente y finalmente las longitudes de los canales que se desprenden de los forámenes.

La recolección de los datos se realizó mediante una inspección visual a través de tres operadores en conjunto. Para obtener los diámetros y longitudes se procedió a fijar un pedazo de papel milimetrado de $2 \mathrm{~cm}$. de alto por $1 \mathrm{~cm}$. de ancho inmediatamente hacia la derecha de la línea mediana para cada mandíbula (Fig. 1). Este papel fue colocado en sentido vertical. Una vez completada esta etapa se obtuvo fotos estandarizadas utilizando una cámara Cibershot Dsc-S75 y un trípode. Se obtuvo 2 imágenes por cada mandíbula, una imagen para los forámenes ubicados superiormente y otra para los forámenes ubicados inferiormente. Se tuvo la precaución de obtener una imagen ortográfica de los forámenes en estudio. Luego de esto se procedió a introducir una lima $\mathrm{k}$ de subserie correspondiente a la lima 0,8 . Se introdujo en cada foramen hasta constatar tope óseo y se llevó a posición el tope de goma. Esta lima luego se colocó sobre un papel milimetrado en el que se encontraba escrito el número de la mandíbula y a que foramen corresponde (Fig. 2).

Finalmente estás imágenes fueron analizadas utilizando el programa Photoshop ${ }^{\circledR}$ Cs 3 Extended. Como este programa mide en pixeles y basándose en el papel milimetrado que se fotografió pegado en la mandíbula, se trasformaron los pixeles a milímetros. Una vez realizada la conversión, se midió con un zoom de $300 \%$ el diámetro de cada forámen (Fig. 3). Para la medición de los forámenes se obtuvo 2 diámetros, uno vertical y otro horizontal. El diámetro considerado para el estudio correspondió al promedio entre ambas mediciones. Para el caso de las imágenes de las limas se utilizó una metodología idéntica constatando la longitud de cada canal, registrando esta información en la tabla.

\section{RESULTADOS}

De 71 mandíbulas, 12 eran totalmente desdentadas, 49 desdentadas parcialmente y 10 con la fórmula dentaria completa (más de 14 piezas). El 97\% de las mandíbulas presentaban uno o más forámenes en relación a las espinas mentonianas. El número máximo de forámenes correspondió a 4 localizados en 5 
mandíbulas, 3 forámenes en 22 mandíbulas, 2 forámenes en 32 mandíbulas, 1 foramen en 10 mandíbulas y ningún foramen en 2 mandíbulas (Tabla I). Para los forámenes encontrados sobre las espinas mentonianas superiores se encontró un foramen en 58 mandíbulas ( $82 \%$ de la muestra), las 13 mandíbulas restantes no presentaron forámenes sobre las espinas mentonianas superiores. Estos forámenes fueron los más constantes dentro de nuestra muestra.

Se observó en 54 mandíbulas (76\% ) forámenes ubicados por inferior a las espinas mentonianas inferiores. Se encontró un máximo de 3 forámenes inferiores en un espécimen (1,4\%), 2 forámenes en 12 mandíbulas (16,9\%), 1 foramen en 37 mandíbulas (52\%) y 17 mandíbulas no presentaron foramen. Respecto a los forámenes ubicados entre las espinas mentonianas superiores (EEMS), entre espinas mentonianas superiores e inferiores (EEMSI) y entre espinas mentonianas inferiores (EEMI) su presencia fue menos constante (Tabla II).

Tabla I. Distribución del número de forámenes.

\begin{tabular}{lcc}
\hline n forámenes & n mandíbulas & \% \\
\hline $\mathbf{0}$ & 2 & 3 \\
$\mathbf{1}$ & 10 & 14 \\
$\mathbf{2}$ & 32 & 45 \\
$\mathbf{3}$ & 22 & 31 \\
$\mathbf{4}$ & 5 & 7 \\
Total & 71 & 100 \\
\hline
\end{tabular}

La cantidad máxima de forámenes encontrados en cada una de estas posiciones por mandíbula fue de 1 foramen.

Respecto al diámetro para los forámenes ubicados por sobre las espinas mentonianas superiores (SEMS) el promedio de diámetro fue de $0,73 \mathrm{~mm}$ (DE 0,31). En el caso de los forámenes inferiores a las espinas mentonianas inferiores (IEMI) el promedio fue de 0,71 mm (DE 0,31). Los diámetros y desviaciones estándar se encuentran en Tabla III.

Finalmente la medición de la longitud de los canales ubicados sobre las espinas mentonianas superiores obtuvo un promedio de 6,77 mm (DE 3,32), los ubicados por inferior a las espinas mentonianas inferiores promediaron 5,5 mm de longitud (DE 2,82). El resto de los promedios, desviación estándar, valores máximos y mínimos se encuentran en la Tabla IV.

Tabla II. Cantidad de especímenes con un foramen ubicado entre las espinas mentonianas superiores (EEMS), entre espinas mentonianas superiores e inferiores (EEMSI) y entre espinas mentonianas inferiores (EEMI).

\begin{tabular}{lcc}
\hline Posición & n mandíbulas & $\begin{array}{c}\text { \% Respecto al total } \\
\text { de la muestra }\end{array}$ \\
\hline EEMS & 4 & 5,6 \\
EEMSI & 18 & 25,4 \\
EEMI & 8 & 11,3 \\
Total & 30 & 42,30 \\
\hline
\end{tabular}

Tabla III. Promedio, desviación estándar, valor máximo y mínimo en milímetros del diámetro de los forámenes ubicados sobre las espinas mentonianas superiores (SEMS), inferiores a las espinas mentonianas inferiores (IEMI), entre las espinas mentonianas superiores (EEMS), entre espinas mentonianas superiores e inferiores (EEMSI) y entre espinas mentonianas inferiores (EEMI)).

\begin{tabular}{lcccc}
\hline Posición del foramen & Diámetro promedio & DE & Valor máximo & Valor mínimo \\
\hline SEMS & 0,73 & 0,31 & 1,7 & 0,3 \\
IEMI & 0,71 & 0,31 & 1,62 & 0,3 \\
EEMS & 0,58 & 0,055 & 0,63 & 0,5 \\
EEMSI & 0,6 & 0,25 & 1,02 & 0,2 \\
EEMI & 0,84 & 0,45 & 1,6 & 0,35 \\
\hline
\end{tabular}

Tabla IV. Promedio, desviación estándar, valor máximo y mínimo de la longitud en milímetros de los canales ubicados sobre las espinas mentonianas superiores (SEMS), inferiores a las espinas mentonianas inferiores (IEMI), entre las espinas mentonianas superiores (EEMS), entre espinas mentonianas superiores e inferiores (EEMSI) y entre espinas mentonianas inferiores (EEMI)).

\begin{tabular}{lcccc}
\hline Posición del canal & Promedio & DE & Valor máximo & Valor mínimo \\
\hline SEMS & 6,77 & 3,32 & 15 & 1,32 \\
IEMI & 5,5 & 2,82 & 12,57 & 1,5 \\
EEMS & 6,56 & 3,22 & 10,37 & 2,5 \\
EEMSI & 5,14 & 3,02 & 11 & 0,7 \\
EEMI & 5,56 & 3,13 & 8,8 & 1,5 \\
\hline
\end{tabular}




\section{DISCUSIÓN}

Múltiples artículos en la literatura han atribuido la formación de hematomas sublinguales, durante la instalación de implantes oseointegrados, al sangramiento de vasos sanguíneos ubicados en la cara interna del cuerpo mandibular (Mordenfeld et al., 1997; Isaacson; Kalpidis \& Setayesh). Si bien es cierto pueden existir diversas ubicaciones para estos vasos (Tagaya et al.), la más común es en relación a línea mediana encontrándose por superior, entre o inferior a las espinas mentonianas, penetrando la cortical de la cara interna del cuerpo mandibular (McDonnell et al.; Mraiwa et al., 2003).

El contenido de este foramen ha sido foco de controversia. Textos clásicos de anatomía como Rouvière \& Delmas (1999), Testut \& Latarjet (1960), Figún \& Garino (1994) en sus respectivos capítulos de osteología de cabeza no mencionan la presencia de forámenes en la cara interna del cuerpo mandibular. Solo hacen mención a la presencia de vasos sanguíneos que pueden penetrar la cortical ósea en esta zona describiéndolos en los capítulos de angiología. Diversos autores describen el contenido de estos forámenes y canales como elementos vasculares derivados de la anastomosis entre ramas de las arterias sublinguales derecha e izquierda (McDonnell et al.; Darriba \& Mendonca-Caridad, 1997; Givol et al., 2000; Lustig et al., 2003), y también anastomosis de éstas con ramas de la arteria submentoniana, rama de la arteria facial (Kalpidis \& Setayesh; Liang et al., 2004). Otros mencionan la presencia de un paquete neurovascular con fibras derivadas del nervio milohioideo, o que el paquete neurovascular es una prolongación del canal incisivo mandibular e incluso que se trataría de un canal eminentemente nervioso (Sutton, 1974; Madeira et al., 1978; Liang et al., 2005). De todas formas, desde el punto de vista práctico, el elemento vascular es el que cobra mayor importancia en cirugía y trauma maxilofacial. La instalación de implantes oseointegrados, profundización de flanco lingual, genioplastías, fracturas sinfisiarias y parasinfisiarias pueden producir daño y complicaciones con estos vasos.

Los resultados en población chilena son consistentes con la literatura publicada en otros grupos étnicos. McDonnell en una muestra de 314 especímenes de población india y australiana describió la presencia de forámenes en un 99,04\% sobre o a nivel de las espinas mentonianas superiores. En nuestro estudio se encontró la presencia de forámenes en $97 \%$ de las mandíbulas y el $82 \%$ de la muestra presentó foramen sobre las espinas mentonianas superiores, siendo el foramen con mayor prevalencia. Nagar et al. (2001) en un trabajo en población India el 72,45\% de la muestra presentó forámenes sobre o a nivel de las espinas mentonianas superiores. Un estudio realizado por De Vasconcellos et al., 2000 señaló que el $100 \%$ de las mandíbulas presentaba forámenes en relación a las espinas mentonianas. En mandíbulas edéntulas el 60,35\% presentó 1 foramen, el 34,48\% presentó dos forámenes y el $5,17 \%$ presentó tres forámenes. En mandíbulas dentadas el $63,79 \%$ presentó un foramen, el 32,76\% dos forámenes y el $3,45 \%$ tres forámenes. Estos números difieren de nuestros resultados ya que el mayor porcentaje de mandíbulas estudiadas en esta investigación tuvo dos forámenes (45\%), mientras que las que tuvieron un solo foramen correspondieron al $14 \%$ de nuestra muestra.

Con respecto al diámetro y longitud de los forámenes y canales, sobre todo los ubicados superior a las espinas mentonianas superiores (SEMS), presentaban el tamaño suficiente $(0,73 \mathrm{~mm}$ de diámetro y $6,77 \mathrm{~mm}$ de longitud) para alojar un vaso sanguíneo, especialmente arterial, que puede ocasionar un accidente hemorrágico de importancia en intervenciones en la zona lingual mediana mandibular. Lustig et al. realizó un estudio utilizando ultrasonido Doopler demostrando la presencia de vasos con flujo de características arteriales que ingresan por estos forámenes, con un diámetro promedio de $1,41 \pm 0,34 \mathrm{~mm}$ y un flujo sanguíneo promedio de 2,92 $\pm 3,19 \mathrm{ml} / \mathrm{min}$ medido justo antes del ingreso al canal lingual respectivo. Con TC Liang et al. (2006) obtuvieron un diámetro promedio de $0,7 \mathrm{~mm}(\mathrm{DE} 0,3)$ para forámenes ubicados en relación a las espinas mentonianas y un promedio de longitud para el canal de 3,9 mm (DE 1,3). Los diámetros son coincidentes con nuestros resultados, sin embargo la longitud difiere probablemente debido a la metodología utilizada por nosotros que puede haber involucrado espacios medulares más allá del canal propiamente tal.

Debido a la alta prevalencia de forámenes y canales en nuestra muestra se recomienda tenerlos siempre en cuenta en la planificación de intervenciones quirúrgicas que involucren, ya sea el flanco lingual mediano mandibular como el cuerpo mandibular en la zona de la sínfisis.

SOTO, R.; CÁCERES, F. \& GARCÍA, R. Presence and morphometry of foramina and canals in relation to mental spines. Int. J. Morphol., 30(2):417-421, 2012.

SUMMARY: Surgical procedures developed in the anterior mandibular body, such as the installation of osseointegrated implants, genioplasties, lingual edge insights, fractures, etc. may produce mayor complications when these invade vascular structures, with consequent bleeding and sublingual hematoma formation. Within these anatomical elements we find foramina and canals that are located in relation to the mental spine. The objective of this study was to quantify the location, diameter and length of the foramina and canals present superior, inferior and between the upper and lower mental spine in 71 dry mandibles from the Morphology Unit, Universidad de los Andes, which were photographed. Analyzing the pictures with Photoshop ( Cs 3 extended program, the diameter and length of the foramina and canals were measured in pixels and then transformed 
into $\mathrm{mm} .97 \%$ of the jaws observed had at least one foramen, $45 \%$ had two and $31 \%$ three. In relation to location $82 \%$ of the mandibles showed foramina in the bones superior and inferior $76 \%$. The average diameter was $0.73 \mathrm{~mm}$ for superior placement and $0.71 \mathrm{~mm}$ for inferior placement. The average length of the superior canals was $6.77 \mathrm{~mm}$ and $5.5 \mathrm{~mm}$ for those inferior to the mental spine. Due to the high prevalence of foramina and canals in our sample its recommended to keep always in mind when planning surgical procedures that involve either medium mandibular lingual flank as the mandibular body at the symphysis area.

\section{KEY WORDS: Lingual canal; Mental spine.}

\section{REFERENCIAS BIBLIOGRÁFICAS}

Darriba, M. A. \& Mendonça-Caridad, J. J. Profuse bleeding and life-threatening airway obstruction after placement of mandibular dental implants. J. Oral Maxillofac. Surg., 55(11):1328-30, 1997.

De Vasconcellos, H.; De siqueira, A.; De Almeida, G.; Tan, M. \& Barros, P. The anatomy of the lingual foramen canal and its related to the mandibular symphysis. Rev. Chil. Anat., 18(1):4751,2000

Figún, M. \& Garino, R. Anatomía Odontológica funcional y aplicada. Buenos Aires, El Ateneo, 1994.

Flanagan, D. Important arterial supply of the mandible, control of an arterial hemorrhage, and report of a hemorrhagic incident. J. Oral Implantol., 29(4):165-73, 2003.

Givol, N.; Chaushu, G.; Halamish-Shani, T. \& Taicher, S. Emergency tracheostomy following life-threatening hemorrhage in the floor of the mouth during immediate implant placement in the mandibular canine region. J. Periodontol., 71(12):1893-5, 2000

Isaacson, T. J. Sublingual hematoma formation during immediate placement of mandibular endosseous implants. J. Am. Dent. Assoc., 135(2):168-72, 2004.

Kalpidis, C. D. \& Setayesh, R. M. Hemorrhaging associated with endosseous implant placement in the anterior mandible: a review of the literature. J. Periodontol., 75(5):631-45, 2004.

Lagraña, R.; Frank, M.; Camacho Verna, M.; Gauna, F. \& Abalo, M. Forámenes Accesorios Del Hueso Mandibular Humano U niversidad Nacional del Nordeste, Comunicaciones Científicas y Tecnológicas. 2006. Disponible en: http:// www. unne.edu.ar/Web/cyt/cyt2006/03-Medicas/2006-M031.pdf

Liang, H.; Frederiksen, N. L. \& Benson, B. W. Lingual vascular canals of the interforaminal region of the mandible: evaluation with conventional tomography. Dentomaxillofac. Radiol., 33(5):340-1, 2004.
Liang, X.; Jacobs, R. \& Lambrichts, I. An assessment on spiral CT scan of the superior and inferior genial spinal foramina and canals. Surg. Radiol. Anat., 28(1):98-104, 2006.

Liang, X.; Jacobs, R.; Lambrichts, I.; Vandewalle, G.; van Oostveldt, D.; Schepers, E.; Adriaensens, P. \& Gelan, J. Microanatomical and histological assessment of the content of superior genial spinal foramen and its bony canal. Dentomaxillofac. Radiol., 34(6):362-8, 2005.

Lustig, J. P.; London, D.; Dor, B. L. \& Yanko, R. Ultrasound identification and quantitative measurement of blood supply to the anterior part of the mandible. Oral Surg. Oral Med. Oral Pathol. Oral Radiol. Endod., 96(5):625-9, 2003.

Madeira, M. C.; Percinoto, C.; das Graças, M. \& Silva, M. Clinical significance of supplementary innervation of the lower incisor teeth: a dissection study of the mylohyoid nerve. Oral Surg. Oral Med. Oral Pathol., 46(5):608-14, 1978.

McDonnell, D.; Reza Nouri, M. \& Todd, M. E. The mandibular lingual foramen: a consistent arterial foramen in the middle of the mandible. J. Anat., 184(Pt 2):363-9, 1994.

Mordenfeld, A.; Andersson, L. \& Bergström, B. Hemorrhage in the floor of the mouth during implant placement in the edentulous mandible: a case report. Int. J. Oral Maxillofac. Implants, 12(4):558-61, 1997.

Mraiwa, д.; Jacobs, R.; van Steenberghe, D. \& Quirynen, M. Clinical assessment and surgical implications of anatomic challenges in the anterior mandible. Clin. Implant Dent. Relat. Res., 5(4):219-25, 2003.

Nagar, M.; Bhardwaj, R. \& Prakash, R. Accessory lingual foramen in adult Indian mandibles. J. Anat. Soc. India, 50(1):134, 2001.

Rouvière, H. \& Delmas, A. Anatomía Humana. Descriptiva, Topográfica y Funcional. 9ª Ed. Barcelona, Ed. Masson, 1999.

Sutton, R. N. The practical significance of mandibular accessory foramina. Aust. Dent. J., 19(3):167-73, 1974.

Tagaya, A.; Matsuda, Y.; Nakajima, K.; Seki, K. \& Okano, T. Assessment of the blood supply to the lingual surface of the mandible for reduction of bleeding during implant surgery. Clin. Oral Implants Res., 20(4):351-5, 2009.

Testut, L. \& Latarjet, A. Tratado de anatomía humana. $9^{\mathrm{a}}$ ed. Barcelona, Salvat, 1960 . V.2

Dirección para correspondencia:

Dr. Reinaldo Soto Norambuena

Av. San Carlos de Apoquindo 2200

Santiago - CHILE

Email: rsoto@miuandes.cl

Recibido : 20-01-2012 Aceptado: 08-04-2012 